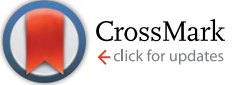

Cite this: RSC Adv., 2017, 7, 3321

\title{
ZnO nanorods assembled with different porphyrins - size-tunable hybrid particles $\uparrow$
}

\begin{abstract}
Jasmin Düring and Franziska Gröhn*
We present a fundamental study on $\mathrm{ZnO}$ nanorod-porphyrin assembly formation in solution, providing the key to novel tunable hybrid assemblies with potential in solar energy conversion. The combination of $40 \mathrm{~nm}$ $\mathrm{ZnO}$ nanorods with ionic porphyrins - meso-tetra(4-carboxyphenyl)porphyrin (TCPP) and meso-tetra-(4sulfonatophenyl)porphyrin (TPPS) - results in the formation of novel well-defined hybrid assemblies which are stable in solution and exhibit an adjustable size up to $500 \mathrm{~nm}$. Structures have been characterized with dynamic light scattering (DLS), transmission electron microscopy (TEM) and absorption and emission spectroscopy. In particular, the combination of both porphyrins with $\mathrm{ZnO}$ in a ternary assembly yields a large stability range in terms of the TCPP/ZnO ratio and may be of significance as a hybrid system for solar cells or photocatalysis.
\end{abstract}

Received 17th November 2016 Accepted 1st December 2016

DOI: $10.1039 / c 6 r a 26964 h$

www.rsc.org/advances

\section{Introduction}

A powerful method to build hybrid nanostructures in solution and on surfaces is self-assembly, that is the formation of organized structures from a disordered system of building blocks. Driving forces can be hydrogen bonding, ionic interactions, $\pi-$ $\pi$ stacking, metal coordination or van der Waals interactions. A large range of structures and potential applications is accessible through the concept of self-assembly. ${ }^{1}$ Yet, in particular for more complex and functional structures, fundamental knowledge on structure formation and control is limited but highly desirable for a broad and targeted exploitation of self-assembly.

Combining semiconducting ZnO nanostructures with porphyrins as light harvesting component through self-assembly may have significant impact in the field of solar energy conversion. ${ }^{2,3}$ With this motivation, we herein fundamentally investigate the assembly formation of $\mathrm{ZnO}$ nanorods with different porphyrins that can interact with the $\mathrm{ZnO}$ surface due to ionic interaction and metal coordination. Both, meso-tetra(4carboxyphenyl)porphyrin (TCPP) and meso-tetra-(4-sulfonatophenyl)porphyrin (TPPS) (Fig. 2) have four functional groups which are able to bind to a $\mathrm{ZnO}$ surface, so that the porphyrin molecules can act as linker and interconnect $\mathrm{ZnO}$ nanorods to into larger organic-inorganic hybrid assemblies. We elucidate influences on the structure formation such as choice and ratio of the building blocks and influence of sonication on the structure formation. Resulting hybrid structures are characterized by dynamic light scattering (DLS), transmission electron microscopy

Department of Chemistry and Pharmacy, Interdisciplinary Center for Molecular Materials, Friedrich-Alexander-University Erlangen-Nürnberg, Egerlandstraße 3, 91058 Erlangen, Germany. E-mail: franziska.groehn@fau.de

$\dagger$ Electronic supplementary information (ESI) available. See DOI: 10.1039/c6ra26964h
(TEM) and spectroscopic methods. The versatility of this modular and supramolecular approach bears high potential for the formation of functional hybrid nanostructures.

\section{Experimental section}

\section{Chemicals}

meso-Tetra(4-carboxyphenyl)porphyrin (TCPP) was obtained from TriPorTech GmbH (Germany), meso-tetra-(4-sulfonatophenyl)porphyrin tetrasodium salt (TPPS, 98.0\%) was received from Fluka and all other chemicals were purchased from SigmaAldrich ${ }^{\circledR}$. All solvents were filtered before use with $0.2 \mu \mathrm{m}$ Millipore PTFE filters to remove dust.

\section{Synthesis of ZnO nanorods}

The ZnO rods were prepared according to literature. ${ }^{4}$ Zinc acetate (0.82 g, $4.5 \mathrm{mmol}$, 99.99\%) was dissolved in $42 \mathrm{~mL}$ methanol and $0.25 \mathrm{~mL}$ water was added. Under vigorous stirring at $60{ }^{\circ} \mathrm{C}$ a solution of $0.49 \mathrm{~g} \mathrm{KOH}(8.75 \mathrm{mmol}, 99.99 \%)$ in $23 \mathrm{~mL}$ $\mathrm{MeOH}$ was added dropwise. During the addition of the $\mathrm{KOH}$ solution, the reaction mixture turned opaque. After $2 \mathrm{~h} 15 \mathrm{~min}$ stirring at $60{ }^{\circ} \mathrm{C}$ the solution was concentrated to $10 \mathrm{~mL}$ at a rotary pump and further stirred at $65{ }^{\circ} \mathrm{C}$ for $22 \mathrm{~h}$. Then the solution was left at rest to decant. The white precipitate was dispersed in $\mathrm{MeOH}$, centrifuged at $3000 \mathrm{rpm}$ for $5 \mathrm{~min}$ and then dispersed in ethanol.

\section{TODA stabilization of ZnO nanorods}

To stabilize the ZnO nanorods in solution [2-(2-(2-methoxyethoxy)ethoxy)acetic acid] (TODA, technical grade) was used. ${ }^{5}$ For this the as prepared $\mathrm{ZnO}$ nanorods were centrifuged at $2000 \mathrm{rpm}$ for $5 \mathrm{~min}$ and then redispersed in an ethanolic TODA solution with a concentration of $c($ TODA $)=1.27 \times 10^{-2} \mathrm{~mol}$ 
$\mathrm{L}^{-1}$. To remove excess TODA the solution was centrifuged at $15000 \mathrm{rpm}$ for $1.5 \mathrm{~h}$ and redispersed in ethanol. The final $\mathrm{ZnO}$ solution, which was used for sample preparation, had a particle concentration of $c(\mathrm{ZnO})=3.77 \times 10^{17}$ particles per L. The $\mathrm{ZnO}$ particles had a length of $38.6 \mathrm{~nm} \pm 15.4 \mathrm{~nm}$ and a diameter of $9.5 \mathrm{~nm} \pm 1.2 \mathrm{~nm}$ (130 particles analyzed with TEM).

\section{Sample preparation}

The stock solution of meso-tetra(4-carboxyphenyl)porphyrin (TCPP) in ethanol had a concentration of $c($ TCPP $)=1.7 \times$ $10^{-4} \mathrm{~mol} \mathrm{~L}^{-1}$. Due to the insufficient solubility of meso-tetra-(4sulfonatophenyl)porphyrin (TPPS) in ethanol at high concentrations, the TPPS stock solution was prepared in filtered methanol with a concentration of $c$ (TPPS) $=9.4 \times 10^{-4} \mathrm{~mol} \mathrm{~L}^{-1}$. The concentration of TPPS in the samples was low enough for the TPPS to be completely dissolved, which was confirmed with absorption spectroscopy. Both porphyrin stock solutions were filtered with hydrophilic $0.2 \mu \mathrm{m}$ Millipore PTFE filters to remove dust. Directly before sample preparation the $\mathrm{ZnO}$ rod stock solution always was sonicated $(860 \mathrm{~W})$ for a few minutes. The light scattering samples were prepared by subsequently mixing filtered ethanol and the desired amounts of the stock solutions via stirring at $990 \mathrm{rpm}$ in dust free glass vials. The $\mathrm{ZnO}$ particle concentration in the sample solutions was kept constant at $c(\mathrm{ZnO})=2.0 \times 10^{16}$ particles per $\mathrm{L}$.

\section{Light scattering}

The measurements were performed in dust free light scattering cuvettes at an instrument which was equipped with a red HeNe laser $(\lambda=632.8 \mathrm{~nm} ; 20 \mathrm{~mW})$, an ALV 5000 correlator with 320 channels (ALV GmbH, Langen, Germany) and an ALV CGS 3 goniometer. The measurements covered an angular range of $30^{\circ} \leq$ $\theta \leq 150^{\circ}$. The intensity autocorrelation function $g_{2}(\tau)$ for each angle was transferred into the electric field autocorrelation function $g_{1}(\tau)$ via the Siegert relation. The electric field autocorrelation function $g_{1}(\tau)$ was successively transformed into the distribution of relaxation times $A(\tau)$ by a regularized inverse Laplace transformation using the program CONTIN developed by S. Provencher. ${ }^{6}$ From the distribution of relaxation times the apparent diffusion coefficient was calculated and via extrapolation to zero scattering vector square the diffusion coefficient was obtained, with which the hydrodynamic radius was received via Stokes-Einstein relation. The error bars were calculated from the error of the extrapolation to zero scattering vector square as described above.

\section{UV-Vis spectroscopy}

Absorption spectra were recorded on a SHIMADZU UV Spectrophotometer (UV-1800) with a slit width of $1.0 \mathrm{~nm}$.

\section{Emission spectroscopy}

Emission spectra were recorded on a FluoroMax-3 from Horiba Scientific using $1 \mathrm{~cm}$ fluorescence quartz cuvettes.

\section{TEM}

Transmission electron microscopy (TEM) images were acquired with a Zeiss EM 900 microscope or a Zeiss EM912 microscope, both operated at $80 \mathrm{kV}$ at magnifications ranging from $20000 \times$ to $250000 \times$. The specimens were prepared by depositing $5 \mu \mathrm{L}$ of the undiluted sample solution onto carbon-coated copper grids, 300 mesh, and immediately remove the solvent via capillary forces.

\section{Results and discussion}

Fig. 1 and 2 depict the building blocks used for supramolecular structure formation in this study. The $\mathrm{ZnO}$ nanorods were prepared according to literature as described in the experimental part ${ }^{4}$ and subsequently coated with [2-(2-(2-methoxyethoxy)ethoxy)acetic acid] (TODA) (Fig. 2A) to provide extended stability in solution. ${ }^{5}$ The characterization of the functionalized ZnO nanorods with transmission electron microscopy (TEM), UV-Vis spectroscopy and dynamic light scattering (DLS) revealed defined $\mathrm{ZnO}$ nanorods with an average size of $38.6 \mathrm{~nm}$ $\times 9.5 \mathrm{~nm}$ (Fig. 1A-C). Transmission electron microscopy (TEM) of a TODA stabilized ZnO sample (Fig. 1A) shows individual rods in coexistence with small bundles of up to 10 rods.

These $\mathrm{ZnO}$ nanorods serve as inorganic building block and can be interconnected by the porphyrins TCPP or TPPS, forming larger hybrid structures, as evident from both, TEM and DLS results in Fig. 3b and c: the carboxylic groups of the TCPP and the sulfonic acid groups of TPPS can bind to the ZnO surface by bridging, ester-like or bidentate binding of the oxygen atoms with zinc atoms. ${ }^{4 d, 7}$ Due to the presence of four functional groups per porphyrin molecules, the porphyrin molecules are able to interconnect the $\mathrm{ZnO}$ nanorods by binding to nanoparticle surfaces of two different nanoparticles yielding larger structures of porphyrin-interconnected $\mathrm{ZnO}$ nanorods. $\$$ Here, the interconnection does not only result in the structural change of the system, but also in a functional change, as the porphyrin can interact with $\mathrm{ZnO}$ electronically, acting as a light harvester and inducing electrons into the $\mathrm{ZnO}$ semiconductor.

\section{Assemblies with TCPP}

First the interconnection of the $\mathrm{ZnO}$ rods by addition of different amounts of TCPP is examined. For this, the ratio of porphyrin and $\mathrm{ZnO}$ is denoted molar ratio $r_{\mathrm{M}}$ and defined as number of porphyrin molecules $N$ (porphyrin) per ZnO nanorod $N(\mathrm{ZnO})$.

$$
r_{\mathrm{M}}=\frac{N(\text { porphyrin })}{N(\mathrm{ZnO})}
$$

Fig. 3a shows dynamic light scattering result of a $\mathrm{ZnO}-\mathrm{TCPP}$ assembly solution. In contrast to the hydrodynamic radius of $R_{\mathrm{H}}$ $=23 \mathrm{~nm}$ of the $\mathrm{ZnO}$ rods (Fig. 1C), the assemblies are larger and show a size of $R_{\mathrm{H}}=129 \mathrm{~nm}$ with a size distribution width of $\sigma=$ 0.22 . Hence, multiple rods have become associated into a larger nanoscale assembly through the porphyrins and these assemblies exhibit a well-defined size in solution. Fig. $3 \mathrm{~b}$ and $\mathrm{c}$

$\$$ The binding of TCPP to the ZnO surface could be determined by centrifugation experiments, where no TCPP could be detected in the supernatant, but all porphyrin was found in the sediment together with the ZnO. 

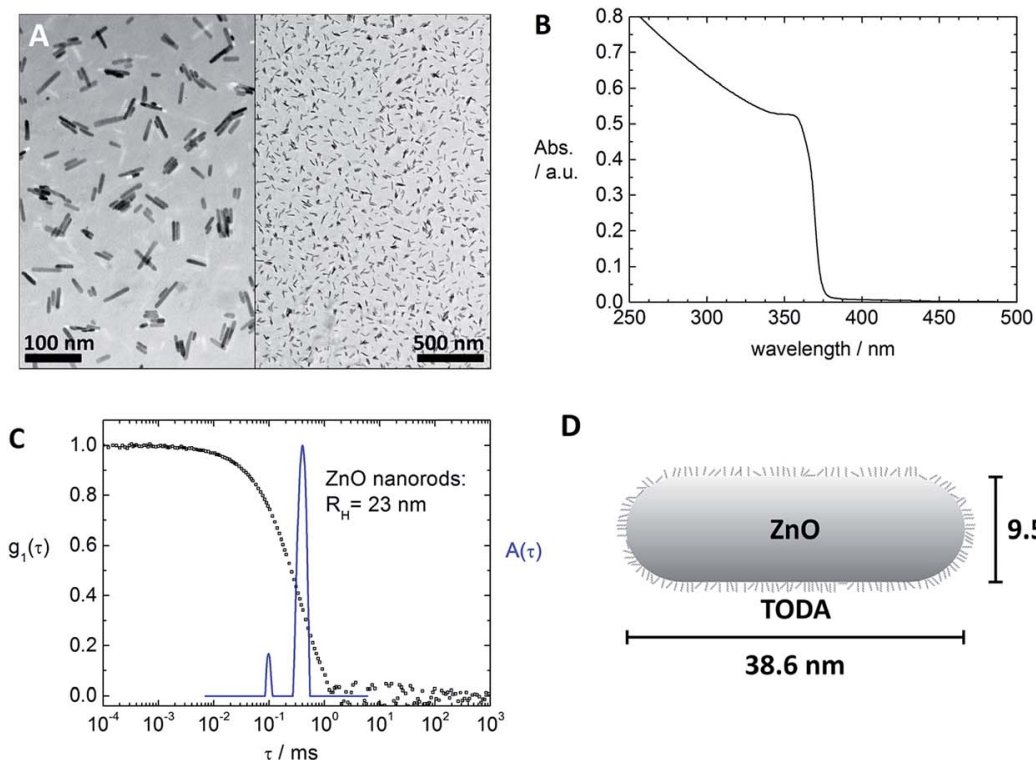

D

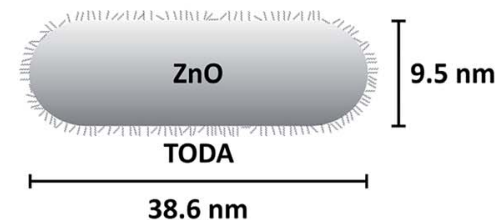

Fig. 1 Inorganic building block for the supramolecular structure formation: characterization of TODA-stabilized ZnO nanorods with TEM (A), UV-Vis spectroscopy $(B)$, dynamic light scattering: electric field autocorrelation function $g_{1}(\tau)$ and distribution of relaxation times $A(\tau)$ at a scattering angle of $\theta=90^{\circ}$ (C). Schematic representation of a TODA-stabilized ZnO nanorod (D) (not to scale).

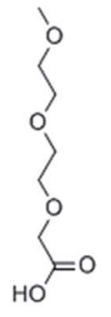

A<smiles></smiles>

B

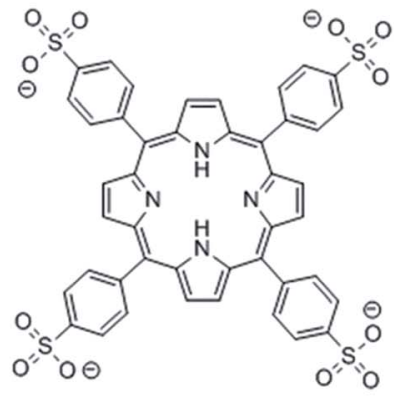

C

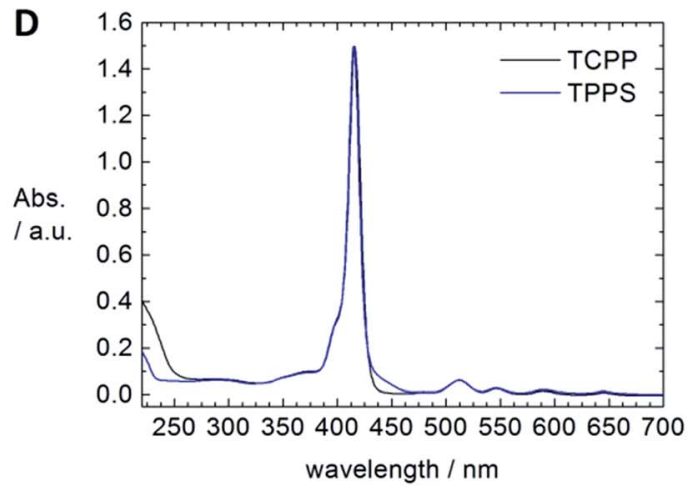

Fig. 2 Organic building blocks for the supramolecular structure formation: structure of 2-(2-(2-methoxyethoxy)ethoxy)acetic acid (TODA) (A), structure of the meso-tetra(4-carboxyphenyl)porphyrin (TCPP) (B), structure of the meso-tetra-(4-sulfonatophenyl)porphyrin (TPPS) (C) and UV-Vis spectra of the two porphyrins in ethanol (D).

visualize these assemblies by TEM, again demonstrating the association of multiple ZnO nanorods. The TEM images show anisotropic assemblies, where due to the low electron contrast of organic material only the $\mathrm{ZnO}$ rods are visible. The overall assembly sizes are in good agreement with the DLS measurement.

Considering both, DLS and TEM in conjunction, it is important to note that the hydrodynamic radius $R_{\mathrm{H}}$ as obtained 
a)
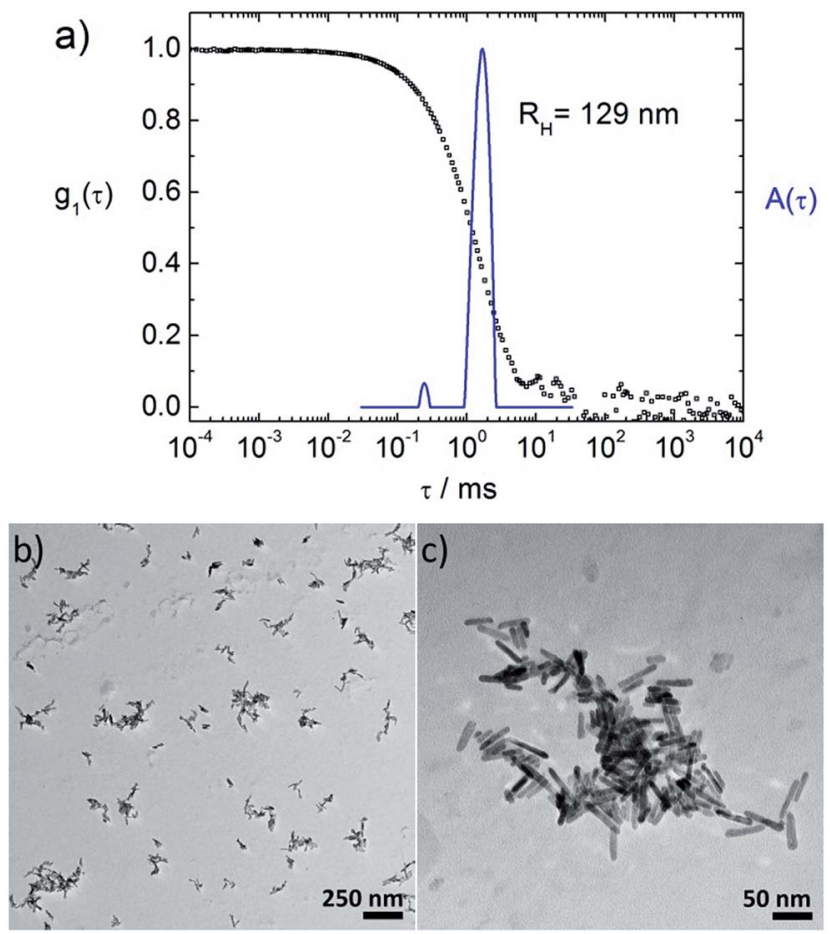

Fig. 3 Characterization of a $\mathrm{ZnO}-\mathrm{TCPP}$ assembly sample with (a) dynamic light scattering: electric field autocorrelation function $g_{1}(\tau)$ and distribution of relaxation times $A(\tau)$ at a scattering angle of $\theta=80^{\circ}$; (b) and (c) TEM pictures of the same ZnO-TCPP assembly sample: (b) overview over several assemblies, (c) magnification of one $\mathrm{ZnO}-\mathrm{TCPP}$ assembly (sample with $c(\mathrm{ZnO})=2 \times 10^{16}$ particles per $\mathrm{L}$, and $r_{\mathrm{M}}(\mathrm{TCPP} / \mathrm{ZnO})=122$ )

in DLS represents the radius of a diffusion equivalent sphere, that is, a dynamic property. On its own, it does not give information on the multiple length scales of particles with anisotropic shapes and/or internal structure but is a good measure of the overall dimension, the width of the size distribution, possible coexistence of multiple sizes, all directly measured in solution with the measurement covering a large particle number. Complementary, TEM visualizes the structure "fully" while one has to keep in mind that the sample is dried on a twodimensional surface which can cause significant structural changes to the solution structure. Thus, it is best to view both very different methods in combination. In front of this background, TEM and DLS are in good agreement for all investigated samples presented in this study; that is, it was possible to image individual assemblies in TEM.

The assembly formation can also be followed spectroscopically. Comparing UV-Vis spectra of a TCPP and a ZnO-TCPP assembly solution, a red shift of $3 \mathrm{~nm}$ from $416 \mathrm{~nm}$ to $419 \mathrm{~nm}$ is observed (Fig. 4a and b), which is understood as the upright binding of TCPP with one or two carboxyl-groups to the surface of $\mathrm{ZnO}{ }^{4 d, 7 a, c, \boldsymbol{d}, \mathbf{8}} \mathrm{A}$ flat face-to-face like conformation of the porphyrin would result in the metalation of the porphyrin cavity, which would lead to a shift of the Soret band to $425 \mathrm{~nm}$ and the presence of only two Q bands instead of four. These spectral changes are indeed observed, but only after an aging time of several days (Fig. S1 $\dagger$ ). Therefore the initial assembly formation is driven by the upright binding of TCPP to the ZnO surface, which leads to the interconnection of several $\mathrm{ZnO}$ nanorods. The comparison of emission spectra of the porphyrin solution and a fresh assembly solution shows a quenching of the TCPP fluorescence in presence of $\mathrm{ZnO}$, as can be seen in Fig. 4c. This quenching is understood as electronic interaction of the TCPP with the ZnO nanorods and can be exploited in solar energy conversion. ${ }^{3 a, 4 d, 9}$

It is then of interest whether the assembly sizes are tunable by variation of the molar ratio $r_{\mathrm{M}}(\mathrm{TCPP} / \mathrm{ZnO})$. The sizes increase with increasing $r_{\mathrm{M}}$ in a way depicted in Fig. 5 . With very small $r_{\mathrm{M}}$ $(<10)$ hardly any interconnection takes place and the assemblies
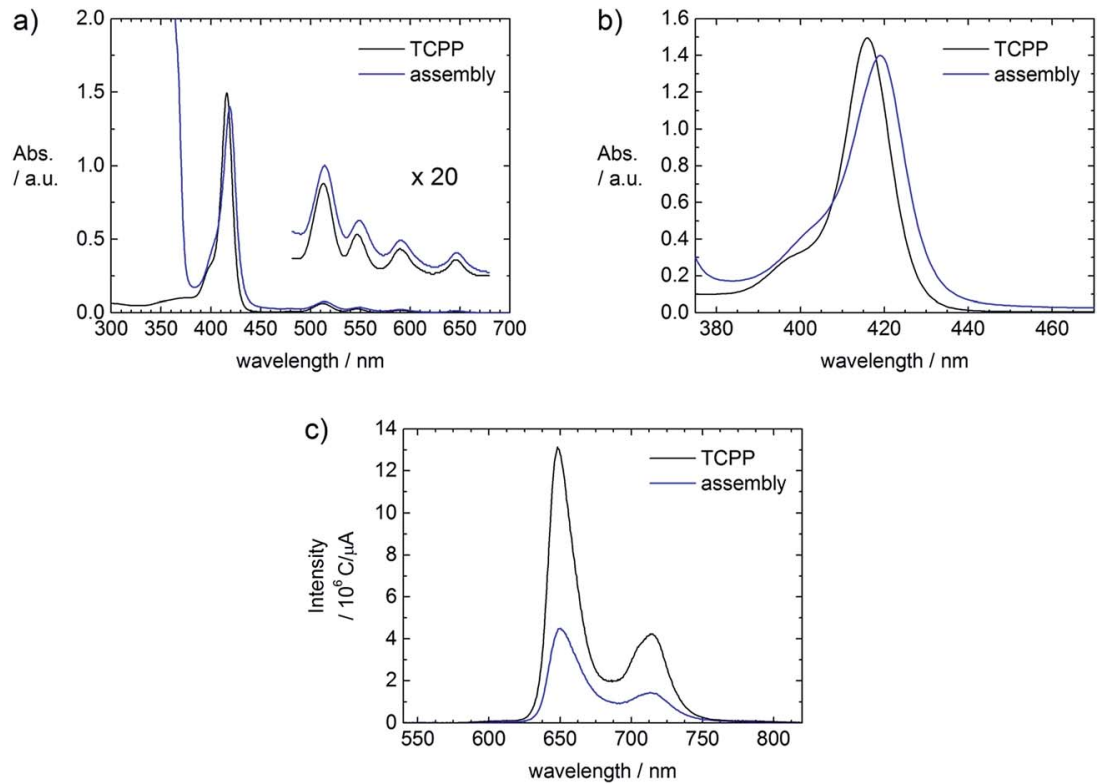

Fig. 4 UV-Vis and emission spectra of TCPP and fresh ZnO-TCPP assemblies in ethanolic solution. (a) and (b) UV-Vis, $r_{M}=106, c(T C P P)=3.3 \times$ $10^{-6} \mathrm{~mol} \mathrm{~L}^{-1}$ in both samples; (c) emission, $\lambda_{\text {exc }}=420 \mathrm{~nm}, r_{\mathrm{M}}=150, c(T C P P)=5.0 \times 10^{-6} \mathrm{~mol} \mathrm{~L}^{-1}$ in both samples. 


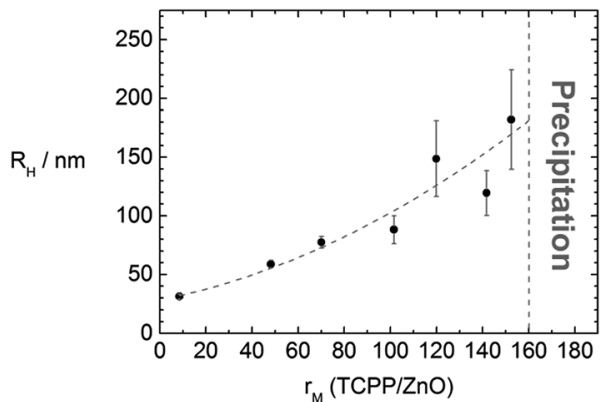

Fig. 5 Dependence of the hydrodynamic radius of the $\mathrm{ZnO}-\mathrm{TCPP}$ assemblies on the molar ratio $r_{M}(T C P P / Z n O)$ with line to guide the eye.

are only slightly larger than the $\mathrm{ZnO}$ nanorods. For molar ratios $r_{M}>160$ the samples precipitate and also for much larger ratios (tested to up to $r_{M}=20000$ ) no stable assemblies can be obtained. For molar ratios $r_{M} \leq 160$ stable assemblies form in solution which show only minor growth within a time range of few days. The large standard deviation, as can be seen in Fig. 5, might be due to a kinetically controlled assembly formation.

As Zn-TCPP has better electronic properties for DSSCs, the same experiments were carried out with $\mathrm{Zn-TCPP}$ in comparison. Zn-TCPP behaves differently from free base TCPP: assemblies with $\mathrm{Zn}$-TCPP are larger than with free base TCPP at the same molar ratios. Furthermore they are not stable, but continue growing until they precipitate, whereby this growth is faster at higher molar ratios. A possible reason for this behavior may lie in the strong stacking of metalized TCPP. Thus, with this additional interaction the stability window for stable assembly formation is narrower than with free base TCPP, leading to larger assemblies and time dependent growth.

\section{Assemblies with TPPS}

To gain a deeper insight into the general binding and interconnection behavior of porphyrin and $\mathrm{ZnO}$, also the meso-tetra(4-sulfonatophenyl)porphyrin (TPPS) was used for assembly formation. TPPS has four sulfonate groups instead of carboxyl groups and can also bind to the ZnO surface. With DLS and
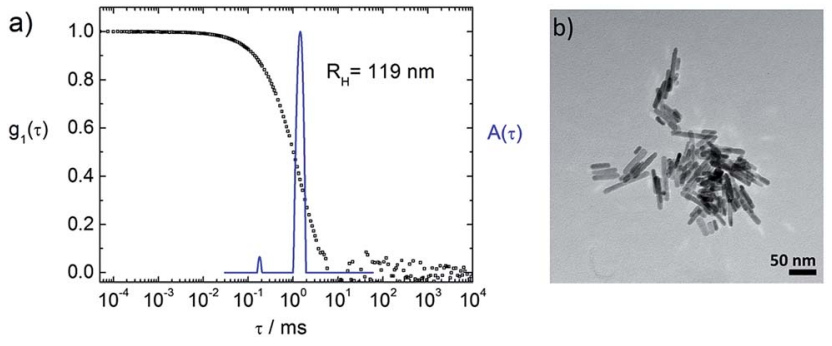

Fig. 6 Characterization of a $\mathrm{ZnO}$-TPPS assembly sample with (a) dynamic light scattering: electric field autocorrelation function $g_{1}(\tau)$ and distribution of relaxation times $A(\tau)$ at a scattering angle of $\theta=90^{\circ}$; (b) TEM on the same ZnO-TPPS assembly sample: magnification of one $\mathrm{ZnO}-\mathrm{TPPS}$ assembly (sample with $c(\mathrm{ZnO})=2 \times 10^{16}$ particles per $\mathrm{L}$, and $\left.r_{\mathrm{M}}(\mathrm{TPPS} / \mathrm{ZnO})=57\right)$.

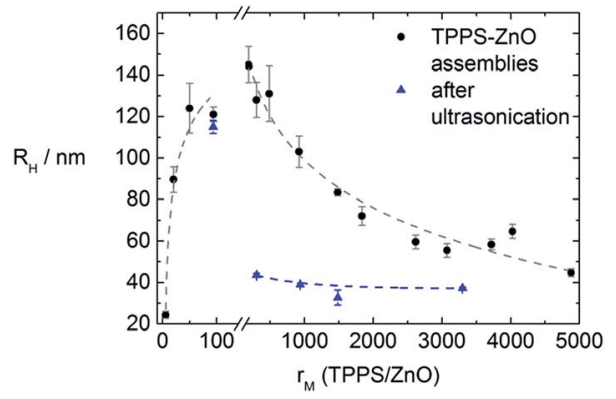

Fig. 7 Size dependence of the supramolecular $\mathrm{ZnO}-\mathrm{TPPS}$ assemblies on the molar ratio of porphyrin to $\mathrm{ZnO}$ (black spheres) with lines to guide the eye. The blue triangles represent the assembly sizes after sonication $\left(c(\mathrm{ZnO})=2.0 \times 10^{16}\right.$ particles per $\left.\mathrm{L}\right)$.

TEM measurements assembly formation can be followed, as can be seen in Fig. 6 .

In analogy to the TCPP-ZnO system also here different molar ratios of porphyrin to $\mathrm{ZnO}$ were tested. The resulting sizes of the assemblies are depicted in Fig. 7.

At molar ratios $r_{\mathrm{M}}<160$ the resulting assembly sizes show a similar dependence on the molar ratio as in the TCPP-ZnO system. At small ratios hardly any size change is observable and the assemblies are larger with larger $r_{M}$. At molar ratios $r_{M}>160$, however, no precipitation occurs in contrast to the TCPP-ZnO system, but the assemblies become smaller, are stable for at least one month and a systematic dependence of size on molar ratio is found. To understand this behavior one has to keep in mind the maximum amount of porphyrin that can attach to each $\mathrm{ZnO}$ nanorod. This value can be calculated considering the average surface of a $\mathrm{ZnO}$ nanorod and the area, each porphyrin molecule requires on a $\mathrm{ZnO}$ surface. The latter area was experimentally determined by Cherian et al. to be $1.2 \mathrm{~nm}^{2}$ per molecule. $^{3 a}$ In a face-to-face conformation TPPS would occupy about $2.3 \mathrm{~nm}^{2}$. In the system here a total porphyrin coverage is reached at 1000 molecules per $\mathrm{ZnO}$ nanorod in case of a porphyrin surface requirement of $1.2 \mathrm{~nm}^{2}$. In case of flat conformation about 500 molecules could fit onto one $\mathrm{ZnO}$ nanorod. However, in this study the $\mathrm{ZnO}$ nanorods are stabilized by TODA, which is, in terms of surface coverage, in competition with the porphyrin. With centrifugation experiments a maximum surface coverage of $r_{\mathrm{M}}(\mathrm{TPPS} / \mathrm{ZnO}) \approx 200$ was determined, which is reasonable considering the presence of TODA. $\S$

Therefore, the assembly size dependence on the molar ratio can be understood in the following way. At ratios $r_{\mathrm{M}}<200$ all porphyrin molecules are immediately attached to the $\mathrm{ZnO}$ surface and interconnect the rods. Small concentration fluctuations can therefore result in different assembly sizes giving quite difficult terms for assembly size prediction.

At larger molar ratios $\left(r_{\mathrm{M}}(\mathrm{TPPS} / \mathrm{ZnO})>200\right)$ the surface of the $\mathrm{ZnO}$ nanorods is saturated with porphyrin. Therefore not every $\mathrm{ZnO}$ nanorod within reach is adsorbed to the assembly, but the most stable assembly size is formed. This allows to predict

$\S$ Only for $r_{M}>200$ TPPS is detectable in the supernatant. 
assembly sizes, whereas a window of $45 \mathrm{~nm}$ to $145 \mathrm{~nm}$ is accessible for tuning of the hydrodynamic radius (for example, Fig. S $2 \uparrow$ shows an example of small assemblies at a large molar ratio of $r_{\mathrm{M}}(\mathrm{TPPS} / \mathrm{ZnO})=4000$, featuring hydrodynamic radii of $\left.R_{\mathrm{H}}=66 \mathrm{~nm}\right)$.

\section{Sonication}

Interestingly, for $r_{M}>200$ it is possible to reduce the assembly sizes upon sonication for $15 \mathrm{~min}$ to hydrodynamic radii between $30 \mathrm{~nm}$ and $45 \mathrm{~nm}$, as can be seen in Fig. 7 (blue triangles) and in Fig. 8. This is not possible for $r_{M}<200$, where the sizes stay nearly constant after sonication. This elucidates the interconnecting behavior of the porphyrin, when every molecule is attached to the $\mathrm{ZnO}$ surface, but the surface is not yet saturated. Even if the $\mathrm{ZnO}$ nanorods are briefly separated by sonication, they immediately become reconnected by the porphyrin. Only once surface saturation with TPPS is reached, the assemblies can become smaller upon sonication, as both surfaces of two freshly separated $\mathrm{ZnO}$ nanorods can be stabilized by free TPPS and therefore do not necessarily aggregate again.

The sonicated samples for $r_{M}>200$ show narrow size distributions (distribution width $\sigma<0.2$ ) and long term stability. Most importantly, the amount of porphyrin can be chosen freely within a wide concentration range and does not determine the assembly size after the sonication.

\section{Absorption spectroscopy}

The spectroscopic investigation yields similar results as in the TCPP system. For molar ratios $r_{M}<200$ a red shift of $2 \mathrm{~nm}$ of the Soret band $\left(\lambda_{\max }(\right.$ TPPS $)=415 \mathrm{~nm}, \lambda_{\max }($ assembly $\left.)=417 \mathrm{~nm}\right)$ can be observed in the fresh assembly. This corresponds to upright binding of the TPPS to the ZnO surface. ${ }^{\boldsymbol{4}, \boldsymbol{7 a}, \mathbf{8}}$ For higher molar ratios $\left(r_{M}>200\right)$, not every porphyrin molecule can simultaneously attach to the surface. Therefore no initial shift is observed in the fresh sample. However, with time a shoulder at higher wavelengths evolves in the Soret band and the intensity ratio of the $\mathrm{Q}$ bands changes, as can be seen in Fig. 9.
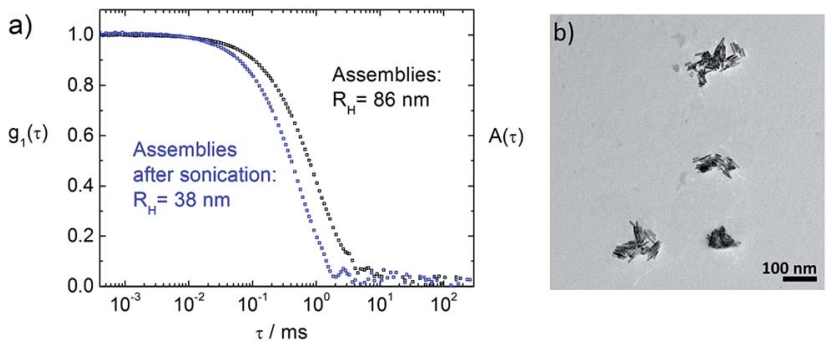

Fig. 8 Investigation of sonicated $\mathrm{ZnO}-\mathrm{TPPS}$ samples: (a) dynamic light scattering: electric field autocorrelation functions $g_{1}(\tau)$ at scattering angles of $\theta=90^{\circ}$. Black curve: $\mathrm{ZnO}-\mathrm{TPPS}$ assemblies with $r_{\mathrm{M}}(\mathrm{TPPS} / \mathrm{ZnO})=1500$, blue curve: same assembly solution after sonication for $15 \mathrm{~min}$, both with $c(\mathrm{ZnO})=2 \times 10^{16}$ particles per $\mathrm{L}$. (b) TEM of a comparable $\mathrm{ZnO}-\mathrm{TPPS}$ sample after sonication.
These time dependent changes can be understood in the way that the TPPS is able to be metalized by the $\mathrm{ZnO}$, forming $\mathrm{Zn}$ TPPS. For $r_{\mathrm{M}}=1500$ this metalation takes place within several hours (Fig. 9). At such a high molar ratio it is impossible for all TPPS molecules to be attached to the ZnO surface. Centrifugation experiments prove that after a certain time free $\mathrm{Zn}$-TPPS is in solution, which means that it is possible for the TPPS to get metalized at the $\mathrm{ZnO}$ surface and subsequently diffuse away again. This gives a hint towards a dynamic attachment and diffusion process of the TPPS at the ZnO surface, facilitated by the sulfonate groups of the porphyrin. The different absorption behavior of TPPS to $\mathrm{ZnO}$ in contrast to a porphyrin featuring a $\mathrm{COOH}$ group was described elsewhere. ${ }^{2 c}$ The time that is necessary to metalize TCPP is another indication of the different behavior of TPPS and TCPP (Fig. S1 $\dagger$ ). The carboxyl groups of TCPP bind to the ZnO surface more strongly making it geometrically more difficult for TCPP to align cofacially to the surface and thereby become metalized. Thus the metalation of TCPP with zinc ions from the ZnO surface takes much longer (several days, Fig. S1†) than the formation of $\mathrm{Zn}$-TPPS (several hours, Fig. 9). Despite the obvious "feeding" of the porphyrin on the $\mathrm{ZnO}$, in TEM measurements no structural change of the $\mathrm{ZnO}$ nanorods is observable. Considering the number of $\mathrm{Zn}$ ions in one $\mathrm{ZnO}$ nanorod, this can easily be understood. Even at a molar ratio of $r_{\mathrm{M}}=1500$ porphyrin molecules per $\mathrm{ZnO}$ nanorod, only $1.4 \%$ of the $\mathrm{Zn}$-ions could be taken out of the nanorod structure, which is negligible. It is also possible to circumvent this attack of the porphyrin on the $\mathrm{ZnO}$. By adding 100 -fold excess of $\mathrm{ZnCl}_{2}$ to the porphyrin solution, metalized $\mathrm{Zn}$-TPPS can be formed. With this Zn-TPPS the same assembly properties were found as with the free base porphyrin. As the metalized porphyrin can only bind with the sulfonate groups, this result also indicates that the upright binding of the porphyrin to the $\mathrm{ZnO}$ surface is structure determining.

\section{Emission spectroscopy}

Emission spectroscopy also indicates interaction of $\mathrm{ZnO}$ with metalized $\mathrm{Zn}$-TPPS. $\uparrow$ At low molar ratios $\left(r_{\mathrm{M}}<200\right)$, where every porphyrin molecule is attached to the $\mathrm{ZnO}$, a distinct quenching of the porphyrin emission can be observed (Fig. 10a). For high molar ratios $\left(r_{\mathrm{M}} \approx 1000\right)$, the emission intensity of the assembly solution is markedly higher than the Zn-TPPS solution of the same concentration, as can be seen in Fig. 10b. At the same time the emission intensities decrease with increasing porphyrin concentration, when the samples are excited with $420 \mathrm{~nm}$ (Fig. 10c).

At high concentrations porphyrins tend to aggregate in solution, as can be followed with UV-Vis spectroscopy. This behavior is even stronger for metalized porphyrins. The aggregation leads to a quenching of the fluorescence, which decreases with decreasing concentration, as also the stacking tendency becomes smaller with lower concentration. Upon

I For emission experiments metalized $\mathrm{Zn}$-TPPS was chosen to simplify and better understand the emission spectra. Otherwise even in fresh assembly samples with TPPS a mixture of TPPS and metalized $\mathrm{Zn}$-TPPS is visible in the spectra. 

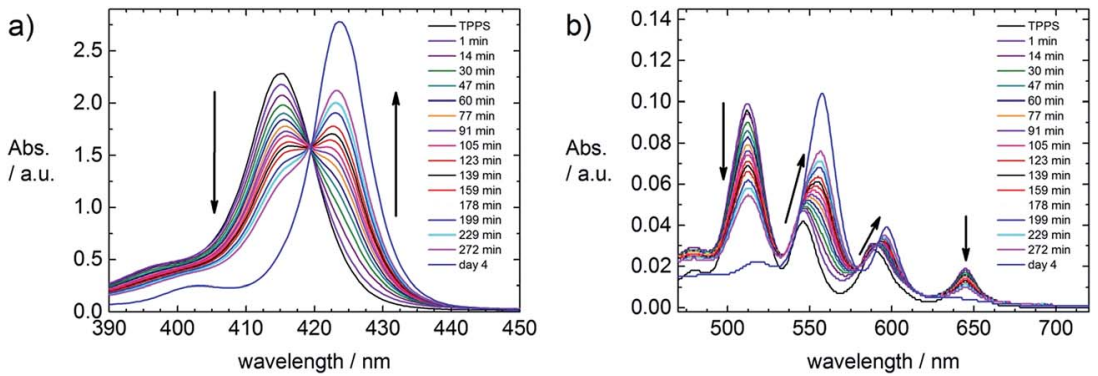

Fig. 9 Time dependent UV-Vis spectra of a ZnO-TPPS sample with $r_{\mathrm{M}}=1500, c(\mathrm{ZnO})=2.0 \times 10^{16}$ particles per L and $c($ TPPS $)=5.1 \times$ $10^{-5} \mathrm{~mol} \mathrm{~L}^{-1}$. (a) Soret bands, (b) $\mathrm{Q}$ bands.
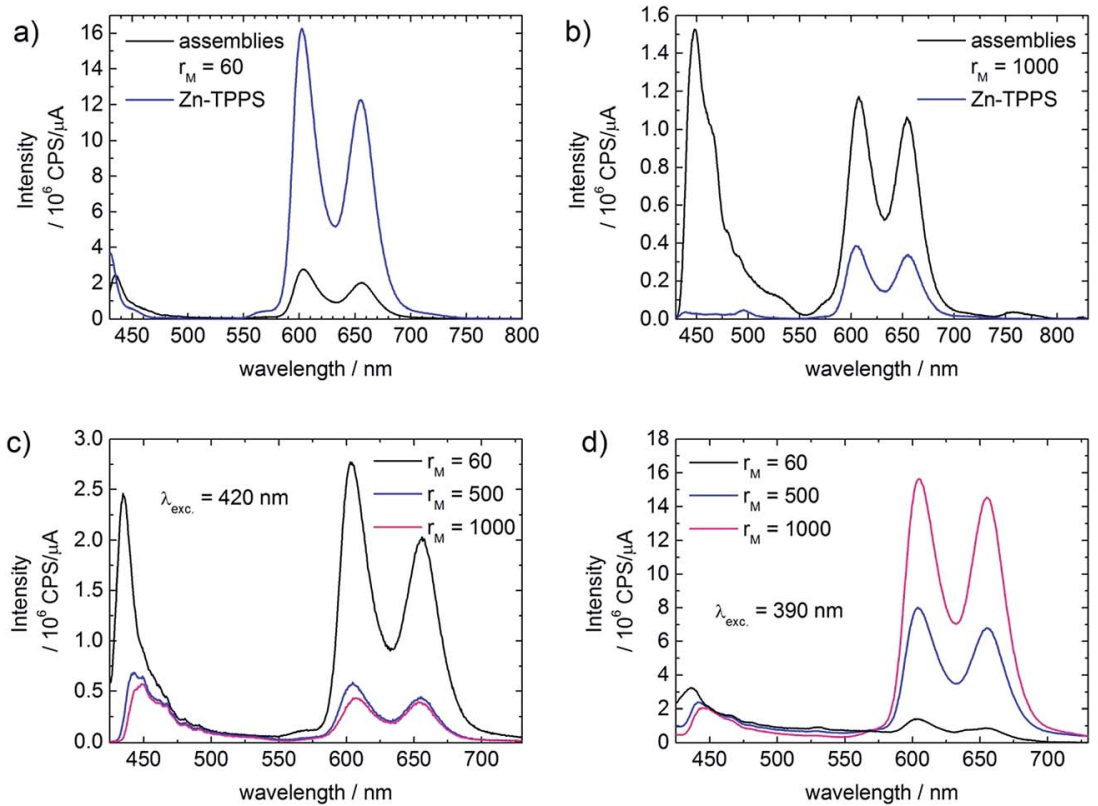

Fig. 10 Emission spectra of different $\mathrm{ZnO}-\mathrm{Zn}$-TPPS assembly samples. (a) and (b) In comparison with $\mathrm{Zn}-\mathrm{TPPS}$ solutions of the same concentrations at $\lambda_{\text {exc }}=420 \mathrm{~nm}$. (a) $r_{\mathrm{M}}=60, c(\mathrm{Zn}-\mathrm{TPPS})=2 \times 10^{-6} \mathrm{~mol} \mathrm{~L}^{-1}$, slit width $=3 \mathrm{~nm}$, (b) $r_{\mathrm{M}}=1000, c(\mathrm{Zn}-\mathrm{TPPS})=3.2 \times 10^{-5} \mathrm{~mol} \mathrm{~L}-1$, slit width $=5 \mathrm{~nm}$. (c) and (d) Three different assembly samples with (c) $\lambda_{\text {exc }}=420 \mathrm{~nm}$, slit width $=3 \mathrm{~nm}$ and (d) $\lambda_{\text {exc }}=390 \mathrm{~nm}$, slit width $=5 \mathrm{~nm}$. Black curves: $r_{M}=60, c(Z n-T P P S)=2 \times 10^{-6} \mathrm{~mol} \mathrm{~L}^{-1}$, blue curves: $r_{\mathrm{M}}=500, c(\mathrm{Zn}-\mathrm{TPPS})=1.6 \times 10^{-5} \mathrm{~mol} \mathrm{~L}^{-1}$, pink curves: $r_{\mathrm{M}}=1000, c(\mathrm{Zn}-$ TPPS $=3.3 \times 10^{-5} \mathrm{~mol} \mathrm{~L}^{-1}$. In all assembly samples: $c(\mathrm{ZnO})=2 \times 10^{16}$ particles per $\mathrm{L}$.

addition of $\mathrm{ZnO}$ nanorods at high $\mathrm{Zn}$-TPPS concentrations a part of the porphyrin binds to the $\mathrm{ZnO}$ surface, thereby lowering the overall concentration of free $\mathrm{Zn}$-TPPS in solution. At lower concentration the porphyrin stacks are smaller, which diminishes the self-quenching and increases the fluorescence (Fig. 10b).

Interestingly, when exciting the samples with $390 \mathrm{~nm}$, that is, not in the region of highest absorption, the emission intensities increase with increasing porphyrin concentration, as can be seen in Fig. 10d. This is the exact opposite of what was found with $420 \mathrm{~nm}$ excitation. These findings can be understood by the inner filter effect: due to the strong absorption of the porphyrin at $420 \mathrm{~nm}$, the intensity of the excitation light is not constant throughout the solution (in case of high porphyrin concentration). In the measuring volume that is significant for the detector, the intensity of the excitation light is markedly weakened, leading to a lower overall intensity of the spectrum. This effect becomes stronger with increasing concentration, causing weaker emission intensity in addition to the self-quenching due to stacking of the porphyrin. At $390 \mathrm{~nm}$ excitation light, the absorption of the porphyrin is weak. Therefore the sample is in the measuring volume irradiated with high intensity, leading to a stronger emission compared to $420 \mathrm{~nm}$ excitation.

The ZnO-Zn-TPPS assemblies feature an additional strong emission band at around $448 \mathrm{~nm}$, as can be seen best in Fig. 10b. $\mathrm{Zn}$-TPPS of low concentration also shows a similar band at $430 \mathrm{~nm}$ (Fig. 10a). For Zn-TPPS alone the ratio between this band and the regular porphyrin emission bands stays constant when changing the excitation wavelength (420 nm, $390 \mathrm{~nm})$. However, the ratios between the first band and the porphyrin emission bands in the assembly samples change with different excitation wavelengths (compare Fig. 10b and c). Furthermore in case of higher porphyrin concentration the first band is markedly stronger in case of assembly samples than in the $\mathrm{Zn}$-TPPS 
solutions (Fig. 10b). This is an indication that the first emission band in the assembly samples is not caused by the porphyrin, but presumably by fluorescence of the $\mathrm{ZnO}$. ZnO alone does not show emission at this excitation wavelength, therefore energy transfer from the excited porphyrin to the $\mathrm{ZnO}$ takes place. The lower intensity of the first band in case of $390 \mathrm{~nm}$ excitation can be understood considering the weaker absorption of the porphyrin at this wavelength. With weak excitation the amount of energy that is transferred to the $\mathrm{ZnO}$ is small, whereas at $420 \mathrm{~nm}$ excitation the absorption of the porphyrin is strong and therefore the amount of transferred energy is high.

The first emission band shows a red shift with increasing porphyrin concentration (435 $\mathrm{nm}$ to $448 \mathrm{~nm}$ ). This red shift might be due to solvatochromism or in this case better described as perichromism. ${ }^{\mathbf{1 0}}$ With higher porphyrin concentration the ratio between TODA and $\mathrm{Zn}$-TPPS at the $\mathrm{ZnO}$ surface shifts towards the porphyrin. Defects in the crystal lattice, which are responsible for the $\mathrm{ZnO}$ emission, ${ }^{\mathbf{1 1}}$ are destabilized due to the different surface environment, lowering the HOMO LUMO gap and thereby causing a red shift of the emission band.

\section{Combination of TPPS and TCPP}

Above we have shown the different binding behavior of TPPS and TCPP. With TPPS a very large range of molar ratios is accessible, where the assembly size deceases for increasing $r_{\mathrm{M}}$ when $r_{\mathrm{M}}<$ 200. Additionally, aggregates can be transferred to smaller well defined assemblies with hydrodynamic radii in a range of $30<R_{\mathrm{H}}$ $<40 \mathrm{~nm}$ via sonication. With TCPP only a small range of molar ratios is accessible, as for $r_{M}>160$ the samples precipitate immediately. This is probably due to the strong binding of the carboxyl group to the $\mathrm{ZnO}$ surface. Combining these two porphyrins could therefore lead to a better stability and thus to a larger molar ratio range accessible for TCPP/ZnO. To verify this hypothesis at first ZnO-TPPS assemblies are formed with $r_{\mathrm{M}}=$ 1000. Then these assemblies are treated with an ultrasonic bath for $15 \mathrm{~min}$, to gain small well defined assemblies of $R_{\mathrm{H}} \approx 30$ $40 \mathrm{~nm}$, which are stabilized by excess TPPS (see Fig. 7 and 9, blue curves). After that, TCPP is added to the solution. For $r_{\mathrm{M}}$ (TCPP/ $\mathrm{ZnO})<160$ the same assembly sizes are found as in the pure ZnO-TCPP system. For larger molar ratios, in difference, no immediate precipitation occurs in contrast to the pure $\mathrm{ZnO}-$ TCPP system (Fig. 11a). This holds true up to $r_{\mathrm{M}}(\mathrm{TCPP} / \mathrm{ZnO})<$ 1000 , for larger ratios the samples precipitate within minutes, which is still slower than without TPPS. These results show that the TPPS is able to stabilize the system, enabling a higher loading of TCPP than without the presence of TPPS. In the range $160<$ $r_{\mathrm{M}}(\mathrm{TCPP} / \mathrm{ZnO})<1000$ the samples show a slow growth with time and the distribution widths are broad $(\sigma>0.35)$. By sonication the hydrodynamic radii can be reduced and the assemblies are much better defined $(\sigma<0.35)$.

In the TEM pictures in Fig. 11b and $\mathrm{c}$ an organic layer is visible around the $\mathrm{ZnO}$ nanorods, which stems from the high overall porphyrin load, that is accessible with the porphyrin mixture approach.

As TCPP in combination with $\mathrm{ZnO}$ is known to be a good dye sensitizer for DSSC, the investigation of this system and
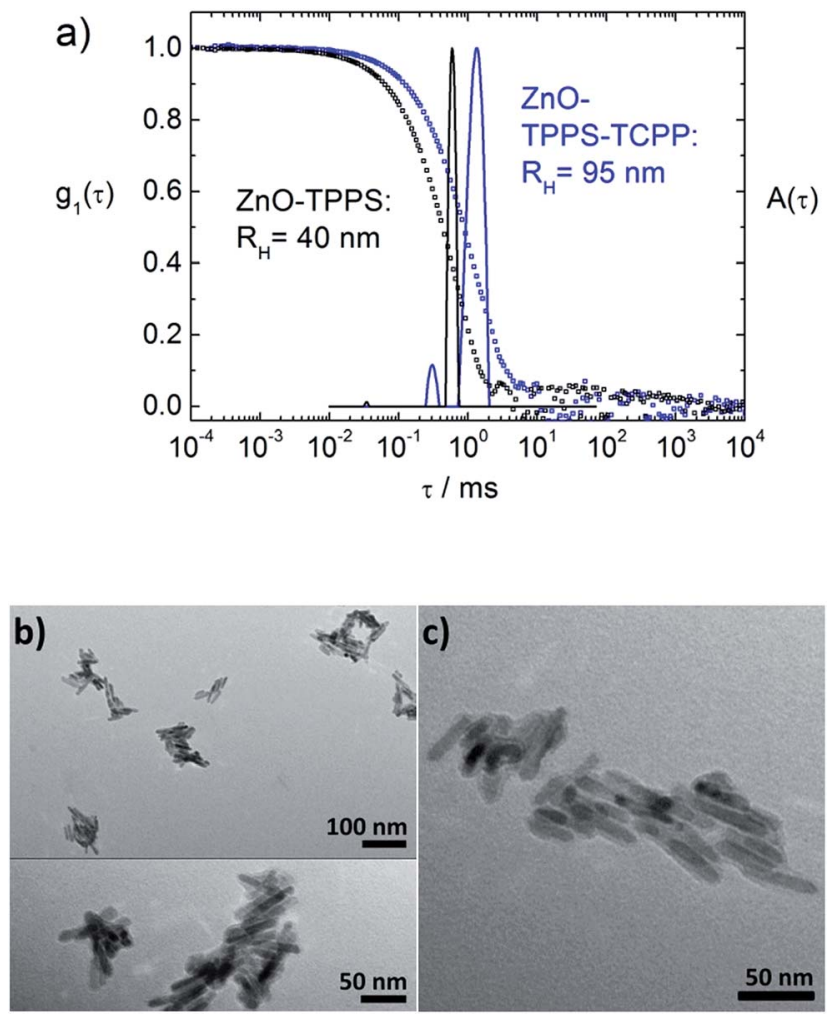

Fig. 11 (a) Dynamic light scattering on $\mathrm{ZnO}-\mathrm{TPPS}$ and $\mathrm{ZnO}-\mathrm{TPPS}-$ TCPP: black: ZnO-TPPS sample with $r_{\mathrm{M}}(\mathrm{TPPS} / \mathrm{ZnO})=1000$ after sonication; blue: same sample after addition of TCPP with $r_{M}$ (TCPP/ $\mathrm{ZnO})=480$ and after sonication; electric field autocorrelation functions $g_{1}(\tau)$ and distributions of relaxation times $A(\tau)$ at scattering angles of $\theta=90^{\circ}$; (b) and (c) TEM of a ZnO-TPPS-TCPP assembly sample after sonication for one hour with $r_{M}(T P P S / Z n O)=1000, r_{M}(T C P P /$ $\mathrm{ZnO})=309, R_{\mathrm{H}}=54 \mathrm{~nm}$ and $\sigma=0.13$; (b) top: overview of several assemblies, bottom: magnification of an assembly section with an organic layer visible; (c) larger magnification of an assembly of the same sample, where the organic layer is visible.

especially the increase of the stability window of TCPP with ZnO by adding another porphyrin that also interacts electronically with $\mathrm{ZnO}$ could be of high relevance in the field of solar energy conversion. In this context it should again be pointed out that all experiments are also possible with the metalized $\mathrm{Zn}$-TPPS. Combining two porphyrins and especially a free base and a metalized porphyrin enhances the absorption coverage of the solar spectrum. Due to the different HOMO-LUMO levels of metalized and free base porphyrin the excited state of the $\mathrm{Zn}$ porphyrin can pass to the free base porphyrin. Furthermore charge recombination can be diminished, as the energetic cascade helps to separate electrons and holes. ${ }^{12}$

\section{Conclusion}

In summary, we have presented the assembly formation of $\mathrm{ZnO}$ nanorods with the two porphyrins TCPP and TPPS and their mixture, as shown in Scheme 1. With TCPP, stable hybrid assemblies form for porphyrin/zinc oxide ratios $r_{M}<160$. With TPPS, assembly formation is possible over a wide range of molar 


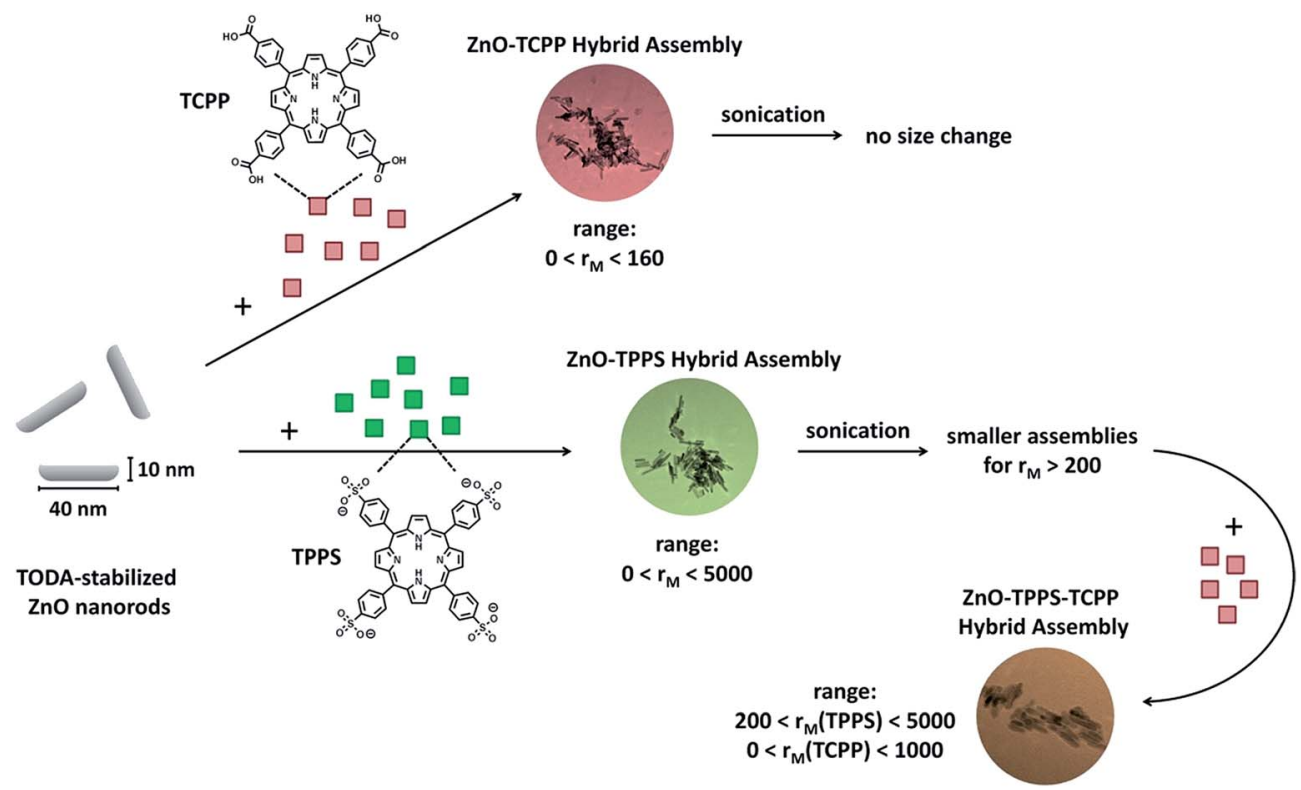

Scheme 1 Schematic representation of the assembly formation of $\mathrm{ZnO}$ nanorods with TCPP and TPPS

ratios $\left(0 \leq r_{\mathrm{M}} \leq 5000\right)$, where the assembly size is adjustable via $r_{\mathrm{M}}$. UV-Vis absorption and emission spectroscopy confirm interaction of both porphyrins with the $\mathrm{ZnO}$ nanorods. With time, the free base porphyrins become metalated and form $\mathrm{Zn}$ porphyrins. For TCPP this process is slow and takes several days, while it is much faster with TPPS taking only several hours, which indicates a more dynamic binding of TPPS to ZnO. In the TPPS-ZnO system it is possible to decrease the assembly sizes to $30<R_{\mathrm{H}}<45 \mathrm{~nm}$ via sonication forming long-term stable hybrid assemblies with narrow size distributions. More complex nanostructures are assembled using a combination of both TCPP and TPPS, resulting in an increased stability range of $r_{\mathrm{M}}(\mathrm{TCPP} / \mathrm{ZnO}) \leq 1000$. Again, upon sonication the sizes of the assemblies can be reduced and the size distributions narrowed (for $\left.150<r_{\mathrm{M}}(\mathrm{TCPP} / \mathrm{ZnO})<1000\right)$. Thus, a large ratio range of porphyrin to $\mathrm{ZnO}$ is accessible by combining the two different porphyrins TCPP and TPPS, with both interacting with the ZnO nanorods. Especially the metalated $\mathrm{Zn}$-TPPS in combination with the free base TCPP could be of high relevance for solar energy conversion, due to enhanced absorption coverage of the solar spectrum and beneficial electronic levels allowing for efficient charge separation. Hence, the comprehension of these porphyrin-ZnO composite assemblies gained herein may contribute significantly to the application of such selfassembled more-component systems in various applications.

\section{Acknowledgements}

Financial support of Deutsche Forschungsgemeinschaft (DFG), the Interdisciplinary Center for Molecular Materials (ICMM, University Erlangen-Nürnberg) and Solar Technologies go Hybrid (SolTech) is gratefully acknowledged.

\section{References}

1 (a) J. Opel, M. Hecht, K. Rurack, J. Eiblmeier, W. Kunz, H. Cölfen and M. Kellermeier, Nanoscale, 2015, 7, 17434; (b) Y. Peng, A.-W. Xu, B. Deng, M. Antonietti and H. Cölfen, J. Phys. Chem. B, 2006, 110, 2988; (c) D. Hentrich, M. Junginger, M. Bruns, H. G. Börner, J. Brandt, G. Brezesinski and A. Taubert, CrystEngComm, 2015, 17, 6901; (d) K. Gorna, R. Muñoz-Espí, F. Gröhn and G. Wegner, Macromol. Biosci., 2007, 7, 163; (e) R. MuñozEspí, A. Chandra and G. Wegner, Cryst. Growth Des., 2007, 7, 1584; $(f)$ M. Antonietti, M. Niederberger and B. Smarsly, Dalton Trans., 2008, 18; (g) R. J. Macfarlane, B. Lee, M. R. Jones, N. Harris, G. C. Schatz and C. A. Mirkin, Science, 2011, 334, 204; (h) X. Liang, L. Yongjun and L. Yuliang, Progress in Chemistry, 2014, 26, 487; (i) X. Li, L. Yang, C. Qin, F.-H. Liu, L. Zhao, K.-Z. Shao and Z.-M. Su, $R S C A d v .$, 2015, 5, 59093; (j) K. W. Tan, B. Jung, J. G. Werner, E. R. Rhoades, M. O. Thompson and U. Wiesner, Science, 2015, 349, 54; $(k)$ M. Jiang, J. A. Kurvits, Y. Lu, A. V. Nurmikko and R. Zia, Nano Lett., 2015, 15, 5010; (l) Y. Min, M. Akbulut, K. Kristiansen, Y. Golan and J. Israelachvili, Nat. Mater., 2008, 7, 527; (m) K. Zhang, M. Jiang and D. Chen, Prog. Polym. Sci., 2012, 37, 445; (n) S. Chen, S.-X. Cheng and R.-X. Zhuo, Macromol. Biosci., 2011, 11, 576.

2 (a) D. Shao, H. Sun, J. Gao, G. Xin, M. A. Aguilar, T. Yao, N. Koratkar, J. Lian and S. Sawyer, Nanoscale, 2014, 6, 13630; (b) Y.-G. Lin, Y.-K. Hsu, Y.-C. Chen, L.-C. Chen, S.-Y. Chen and K.-H. Chen, Nanoscale, 2012, 4, 6515; (c) Y. Sivalingam, G. Magna, E. Martinelli, G. Pomarico, A. Catini, A. D'Amico, R. Paolesse and C. Di Natale, The 14th International Meeting on Chemical Sensors, 2012, DOI: 10.5162/IMCS2012/4.1.1; (d) H. Saarenpää, E. Sariola- 
Leikas, A. Pyymaki Perros, J. M. Kontio, A. Efimov, H. Hayashi, H. Lipsanen, H. Imahori, H. Lemmetyinen and N. V. Tkachenko, J. Phys. Chem. C, 2012, 116, 2336; (e) Y. Chen, M. Yue, Z.-H. Huang, L.-N. Wang and F. Kang, $R S C$ Adv., 2015, 5, 23174; (f) H. Hakola, A. Pyymaki Perros, P. Myllyperkiö, K. Kurotobi, H. Lipsanen, H. Imahori, H. Lemmetyinen and N. V. Tkachenko, Chem. Phys. Lett., 2014, 592, 47; ( $g$ ) P. Kar, S. Sardar, E. Alarousu, J. Sun, Z. S. Seddigi, S. A. Ahmed, E. Y. Danish, O. F. Mohammed and S. Kumar Pal, Chem.-Eur. J., 2014, 20, 10475; (h) W. Sun, J. Li, G. Mele, Z. Zhang and F. Zhang, J. Mol. Catal. A: Chem., 2013, 366, 84; (i) F. Werner, J.-F. Gnichwitz, R. Marczak, E. Palomares, W. Peukert, A. Hirsch and D. M. Guldi, J. Phys. Chem. B, 2010, 114, 14671; (j) G. Magnaa, Y. Sivalingam, E. Martinelli, G. Pomarico, F. Basoli, R. Paolesse and C. Di Natale, Anal. Chim. Acta, 2014, 810, 86; (k) A. Di Mauro, E. Smecca, A. D'Urso, G. G. Condorelli and M. E. Fragalà, Mater. Chem. Phys., 2014, 143, 977; (l) S. Senthilkumar, R. Hariharan, A. Suganthi, M. Ashokkumar, M. Rajarajan and K. Pitchumani, Powder Technol., 2013, 237, 497.

3 (a) S. Cherian and C. C. Wamser, J. Phys. Chem. B, 2000, 104, 3624; (b) S. K. Das, N. K. Subbaiyan, F. D'Souza, A. S. D. Sandanayaka, T. Hasobe and O. Ito, Energy Environ. Sci., 2011, 4, 707.

4 (a) C. Pacholski, A. Kornowski and H. Weller, Angew. Chem., Int. Ed., 2002, 41, 1188; (b) M. Voigt, M. Klaumünzer, H. Thiem and W. Peukert, J. Phys. Chem. C, 2010, 114, 6243; (c) B. Sun and H. Sirringhaus, Nano Lett., 2005, 12, 2408; (d) A. J. Said, G. Poize, C. Martini, D. Ferry,
W. Marine, S. Giorgio, F. Fages, J. Hocq, J. Bouclé, J. Nelson, J. R. Durrant and J. Ackermann, J. Phys. Chem. C, 2010, 114, 11273.

5 S. Schäfer, R. Srikantharajah, M. Klaumünzer, V. Lobaz, M. Voigt and W. Peukert, Thin Solid Films, 2014, 562, 659.

6 S. Provencher, Comput. Phys. Commun., 1982, 27, 229.

7 (a) W. Tu, J. Lei, P. Wang and H. Ju, Chem.-Eur. J., 2011, 17, 9440; (b) W. W. Tu, Y. T. Dong, J. P. Lei and H. X. Ju, Anal. Chem., 2010, 82, 8711; (c) J. Rochford and E. Galoppini, Langmuir, 2008, 24, 5366; (d) J. Rochford, D. Chu, A. Hagfeldt and E. Galoppini, J. Am. Chem. Soc., 2007, 129, 4655; (e) A. Kathiravan, P. S. Kumar, R. Renganathan and S. Anandan, Colloids Surf., A, 2009, 333, 175; (f) M. Niskanen, M. Kuisma, O. Cramariuc, V. Golovanov, T. I. Hukka, N. Tkachenko and T. T. Rantala, Phys. Chem. Chem. Phys., 2013, 15, 17408.

8 I. Hiromitsu, A. Kawami, S. Tanaka, S. Morito, R. Sasai, T. Ikeue, Y. Fujita and M. Handa, Chem. Phys. Lett., 2011, $501,385$.

9 A. S. Huss, A. Bierbaum, R. Chitta, D. J. Ceckanowicz, K. R. Mann, W. L. Gladfelter and D. A. Blank, J. Am. Chem. Soc., 2010, 132, 13963.

10 C. Reichardt and T. Welton, Solvents and Solvent Effects in Organic Chemistry, Wiley-VCH, Weinheim, 2011, pp. 371393.

11 A. B. Djurisic and Y. H. Leung, Small, 2006, 2, 944.

12 D. M. Guldi, I. Zilbermann, G. Anderson, A. Li, D. Balbinot, N. Jux, M. Hatzimarinaki, A. Hirsch and M. Prato, Chem. Commun., 2004, 726. 